\title{
Effect of biologics in the level of cytokines in the synovial fluid of patients with ankylosing spondylitis
}

\author{
Bon San Koo ${ }^{1}$, Sungsin Jo², Eunji Kwon², Ji Hui Shin ${ }^{2}$, Jin-Wuk Hur ${ }^{3}$, and Tae-Hwan Kim²
}

${ }^{1}$ Department of Internal Medicine, Inje University Seoul Paik Hospital, Seoul; ${ }^{2}$ Department of Rheumatology, Hanyang University Hospital for Rheumatic Diseases, Seoul; ${ }^{3}$ Division of Rheumatology, Department of Internal Medicine, Eulji General Hospital, Eulji University School of Medicine, Seoul, Korea

\author{
Received: October 13, 2018 \\ Revised : January 24, 2019 \\ Accepted: January 31, 2019

\section{Correspondence to} \\ Tae-Hwan Kim, M.D. \\ Department of Rheumatology, \\ Hanyang University Hospital \\ for Rheumatic Diseases, 222-1 \\ Wangsimni-ro, Seongdong-gu, \\ Seoul 04763, Korea \\ Tel: +82-2-2290-9245 \\ Fax: +82-2-2298-8231 \\ E-mail: thkim@hanyang.ac.kr
}

Background/Aims: Biologics are very effective drugs for patients with ankylosing spondylitis (AS). However, there are patients who are not responding to biologics. This study aimed to evaluate the level of tumor necrosis factor $\alpha$ (TNF- $\alpha$ ), interleukin (IL)-23, and IL-17 from synovial fluid in patients with AS and rheumatoid arthritis (RA) and differences of the level of those cytokines according to drugs.

Methods: Synovial fluid was obtained from 34 patients (42 samples) with AS and 45 patients (47 samples) with RA with active arthritis of the knee, and the cytokine levels were measured. The differences in the levels between patients treated with and without biologics (biologics and non-biologics groups, respectively) were analyzed in AS and RA. The correlations between cytokines were examined in the non-biologics and biologics groups.

Results: The TNF- $\alpha$ level in AS was significantly lower than that in RA $(p=0.016)$. The IL-17 and IL-23 levels were not different between AS and RA ( $p=0.409$ and $p$ $=0.562$, respectively). In AS and RA, TNF- $\alpha$, IL-17, and IL-23 showed good correlation among each other in the non-biologics group. However, there was no significant correlation in biologics group. In some patients in the AS group, the IL-17 or IL-23 level was markedly elevated in the biologics group.

Conclusions: Treatment with biologics affects the cytokine profile in inflammatory synovial fluid in patients with both AS and RA. Furthermore, IL-23 and IL-17 cytokine might be an important factor in some patients who are unresponsive to biologics in AS.

Keywords: Spondylitis, ankylosing; Arthritis, rheumatoid; Synovial fluid; Interleukins

\section{INTRODUCTION}

Ankylosing spondylitis (AS) and rheumatoid arthritis (RA) have common features, including chronic inflammation. Systemic RA, which affects joints, involves complex cytokine networks that contribute to the autoimmune processes, and localized high concentrations of cytokines cause synovial proliferation, hyperplasia, and angiogenesis [1]. Meanwhile, AS is characterized by syndesmophytes and axial joint ankylosis, and the prima- ry site of inflammation is the enthesis or subchondral bone marrow with bone marrow edema and inflammatory cell infiltration [2].

Despite the differences in the pathogenesis of AS and RA, the treatment of these diseases overlaps. Several biologic drugs targeting inflammatory cytokines have been recommended in AS and RA [3,4]. Conventional disease-modifying anti-rheumatic drugs (DMARDs), such as sulfasalazine, are prescribed in both AS and RA. Both diseases are associated with increased proinflam- 
matory cytokine expression at the site of inflammation and respond well to biologics. Various studies have demonstrated that tumor necrosis factor- $\alpha$ (TNF- $\alpha$ ) is involved in the proinflammatory processes $[1,5-7]$. For RA, alternative approved biologics, such as those directed against cytotoxic T-lymphocyte-associated protein (CTLA) 4-driven T cells, CD2o-expressing B cells, or the interleukin (IL) 6 receptor, have been used. However, unlike alternative biologics used in RA, secukinumab, an anti-IL-17 monoclonal antibody, has been shown to have significantly reduced the disease activity of AS and has been suggested to reduce radiologic changes [8].

However, even though the therapeutic effect of biologics in AS is good, many patients are not treated with drugs $[9,10]$. Determining which patients are refractory to biologics treatment is a very important issue [11]. Thus, in this study, we analyzed cytokines of synovial fluid, because it is difficult to sample the inflammation site. Additionally, the cytokine profile of the synovial fluid, which depends on the current therapeutic agent, is needed to determine the cause of peripheral arthritis during treatment with TNF- $\alpha$ inhibitor.

The aim of this study was to analyze the level of inflammatory cytokines in the synovial fluid of patients with AS according to the use of biologics, compared with that of patients with RA, and identify cytokines that play a major role in the synovial fluid of patients refractory to therapy in AS.

\section{METHODS}

\section{Patients}

Patients with active arthritis of the knee were enrolled at a rheumatology clinic in a tertiary hospital between September 2016 and March 2018. Joint fluid was obtained from 34 patients (42 samples) with AS and 45 patients (47 samples) with RA according to 2010 American College of Rheumatology/European League Against Rheumatism classification criteria in RA and modified New York criteria in AS $[12,13]$. To exclude noninflammatory arthritis, more than $2,000 / \mathrm{mm}^{3}$ of white blood cells and more than $50 \%$ of polymorphonuclear cells in white blood cells were selected in joint fluid analysis. All patients gave informed consent. The study was performed according to the principles of the Declaration of Helsinki.
The Institutional Review Boards of all involved institutions approved this study (HYUH 2016-07-027).

Clinical information including age, sex, disease duration, C-reactive protein (CRP) and erythrocyte sedimentation rate (ESR) levels, and current arthritis medications, such as nonsteroidal anti-inflammatory drugs (NSAIDs), DMARDs (such as sulfasalazine, methotrexate, leflunomide, and tacrolimus), and biologics (etanercept, adalimumab, infliximab, golimumab, abatacept, tocilizumab), were reviewed from electronic medical records. The Bath Ankylosing Spondylitis Disease Activity Index (BASDAI) was measured in patients with AS [14].

We analyzed the effect of current medications on the cytokine levels in the synovial fluid patients with AS and RA. To evaluate the differences in the cytokine levels due to medication, patients with both AS and RA were divided into two groups: the non-biologics group included patients treated with steroids, NSAIDs, or DMARDs other than biologics, and the biologics group included patients who had been treated with biologics.

We performed further analysis in patients with AS. The correlation between cytokines was examined in the non-biologics and biologics groups to demonstrate the differences in cytokine interactions by biologics agents. The difference in cytokine levels according to the use of drugs such as DMARDs and biologics was also analyzed.

\section{Measurement of cytokines levels}

Synovial fluid obtained by therapeutic arthrocentesis was analyzed using blood cell count including polymorphonuclear and mononuclear cells. The rest of the synovial fluid was processed within an hour of collection. The supernatant was assayed for TNF- $\alpha$, IL-17, and IL-23 (BioLegend, San Diego, CA, USA) with an enzyme-linked immunosorbent assay kit according to the manufacturer's recommendation.

\section{Statistics}

Since the data were not normally distributed, they were expressed as median and interquartile range. The Mann-Whitney $U$ test, the chi-square test, and Fisher's exact test were used for comparisons between groups. A correlation test was conducted between cytokines and inflammatory markers. A p value $<0.05$ was considered statistically significant. All statistical analyses were performed with $R$ statistical language version 3.4.1 (R Foun- 
Table 1. Clinical characteristics and cytokine levels from synovial fluid in patients with ankylosing spondylitis and rheumatoid arthritis

\begin{tabular}{|c|c|c|c|}
\hline Characteristic & $\operatorname{AS}(n=34)$ & $\mathrm{RA}(\mathrm{n}=45)$ & $p$ value \\
\hline Age, yr & $35.2(24.0-46.0)$ & $58.0(45.0-62.0)$ & $<0.001$ \\
\hline Male sex & $23(67.6)$ & $9(20.0)$ & $<0.001$ \\
\hline HLA-B27 positive & $30(88.2)$ & - & \\
\hline Rheumatoid factor positive & $3(8.8)$ & $31(68.9)$ & $<0.001$ \\
\hline Anti-CCP positive & $1(2.9)$ & $31(68.9)$ & $<0.001$ \\
\hline $\mathrm{CRP}, \mathrm{mg} / \mathrm{dL}$ & $3.6(0.8-6.7)$ & $0.8(0.4-2.6)$ & 0.002 \\
\hline $\mathrm{ESR}, \mathrm{mm} / \mathrm{hr}$ & $45.5(12.0-81.0)$ & $40.0(18.0-73.0)$ & 0.804 \\
\hline BASDAI & $4.4(2.5-6.6)$ & - & - \\
\hline Treated with steroid & $17(50.0)$ & $34(75.6)$ & 0.035 \\
\hline Treated with NSAIDs & $29(85 \cdot 3)$ & $43(95 \cdot 6)$ & 0.234 \\
\hline Treated with DMARDs & $16(47 \cdot 1)$ & $44(97.8)$ & $<0.001$ \\
\hline Treated with biologics & $7(20.6)$ & $9(20.0)$ & $>0.999$ \\
\hline Infliximab & $4(11.8)$ & 0 & \\
\hline Etanercept & $2(5 \cdot 9)$ & $2(4 \cdot 4)$ & \\
\hline Adalimumab & $1(2.9)$ & $2(4 \cdot 4)$ & \\
\hline Golimumab & 0 & $1(2.2)$ & \\
\hline Abatacept & o & $3(6.7)$ & \\
\hline Tofacitinib & o & $1(2.2)$ & \\
\hline
\end{tabular}

Values are presented as median (range) or number (\%).

AS, ankylosing spondylitis; RA, rheumatoid arthritis; HLA, human leukocyte antigen; CCP, cyclic citrullinated peptide; CRP, C-reactive protein; ESR, erythrocyte sedimentation rate; BASDAI, Bath Ankylosing Spondylitis Disease Activity Index; NSAID, nonsteroid anti-inflammatory drug; DMARD, disease modifying anti-rheumatic drug.

dation for Statistical Computing, Vienna, Austria).

\section{RESULTS}

\section{Clinical characteristics of patients}

The basic clinical characteristics of the patients are shown in Table 1. The median age of patients with AS was significantly younger than that of patients with RA $(p<0.001)$, and there were more male patients in those with AS group compared with those with RA $(p<0.001)$. Human leukocyte antigen (HLA)-B27 was positive in $88.2 \%$ of patients with AS. Rheumatoid factor and anti-cyclic citrullinated peptide (CCP) positivity presented more in patients with RA compared with those with AS $(p<0.001)$. There was no significant difference in the ESR level between AS and RA; however, the CRP level was elevated in patients with AS compared with those with RA $(p=0.002)$.
There was a difference in current medication between the two diseases. The ratio of patients taking steroids or DMARDs was significantly higher in RA compared with $\operatorname{AS}(p=0.035$ and $p<0.001$, respectively). Patients receiving steroids were common in both groups and $50.0 \%$ in patients with AS. These patients used oral steroids as a temporary bridge therapy for starting DMARDs or for improvement of transient arthritis. The ratio of those taking NSAIDs and biologics was not different between the two diseases $(p=0.234$ and $p=1.000$, respectively). Biologics used in patients with AS were all TNF inhibitors (infliximab, $\mathrm{n}=4$; etanercept, $\mathrm{n}=2$; adalimumab, $\mathrm{n}$ $=1$ ). The biologics used in nine patients with RA were etanercept $(n=2)$, adalimumab $(n=2)$, golimumab $(n=$ 1), abatacept $(n=3)$, and tofacitinib $(n=1)$.

\section{Cytokine levels in synovial fluid between AS and RA}

Table 2 shows the different characteristics of the synovial fluid of patients with AS and RA. The white blood 
Table 2. The different characteristics of synovial fluid between ankylosing spondylitis and rheumatoid arthritis

\begin{tabular}{lccc}
\hline Characteristic & AS $(\mathrm{n}=42)$ & RA $(\mathrm{n}=47)$ & $p$ value \\
\hline White blood cell, $/ \mathrm{mm}^{3}$ & $8,650(6,700-10,700)$ & $7,800(6,050-9,900)$ & 0.055 \\
Neutrophil, \% & $61.8(57.9-69.2)$ & $65.7(59.5-73.0)$ & 0.125 \\
Monocyte, \% & $7.7(6.6-9.2)$ & $7.8(6.4-9.1)$ & 0.987 \\
Lymphocyte, \% & $27.1(21.2-32.0)$ & $23.9(16.2-30.2)$ & 0.066 \\
Eosinophil, \% & $1.4(0.7-3.0)$ & $1.5(0.8-2.6)$ & 0.786 \\
TNF- $\alpha, \mathrm{pg} / \mathrm{mL}$ & $127.6(94.2-378.2)$ & $336.5(139.4-553.0)$ & 0.016 \\
IL-17, pg/mL & $36.3(18.9-54.1)$ & $45.8(18.0-70.4)$ & 0.409 \\
IL-23, pg/mL & $511.6(329.4-753.9)$ & $459.7(314.3-1211.9)$ & 0.562 \\
\hline
\end{tabular}

AS, ankylosing spondylitis; RA, rheumatoid arthritis; TNF- $\alpha$, tumor necrosis factor- $\alpha$; IL, interleukin.

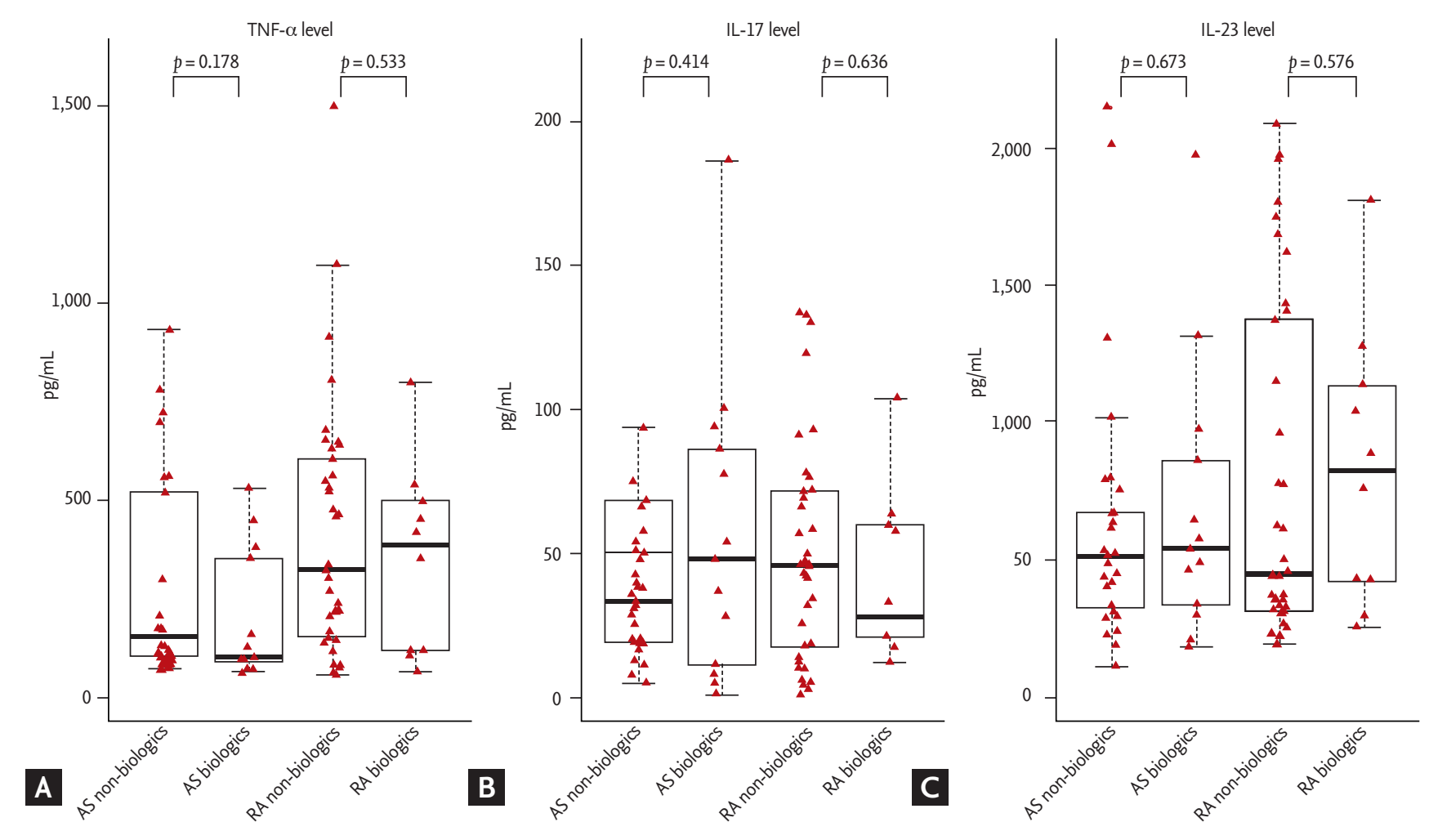

Figure 1. Differences in (A) tumor necrosis factor- $\alpha$ (TNF- $\alpha$ ), (B) interleukin 17 (IL-17), and (C) IL-23 levels between the nonbiologics and biologics groups in patients with ankylosing spondylitis (AS; nonbiologics, $n=29$; biologics, $n=13$ ) and rheumatoid arthritis (RA; nonbiologics, $\mathrm{n}=37$; biologics, $\mathrm{n}=10$ ).

cell count and the percentage of neutrophils, monocytes, lymphocytes, and eosinophils were not different between AS and RA. In terms of cytokines, the level of TNF- $\alpha$ in AS was significantly lower than that in RA ( $p=$ 0.016). IL-17 and IL-23 levels were not statistically significant difference between AS and RA.

\section{Cytokine levels between the non-biologics and biologics group}

The patients with AS and RA were divided into non-biologics and biologics groups to compare the cytokine levels (Fig. 1). The TNF- $\alpha$, IL-17, and IL-23 levels showed no significant difference between the non-biologic and 


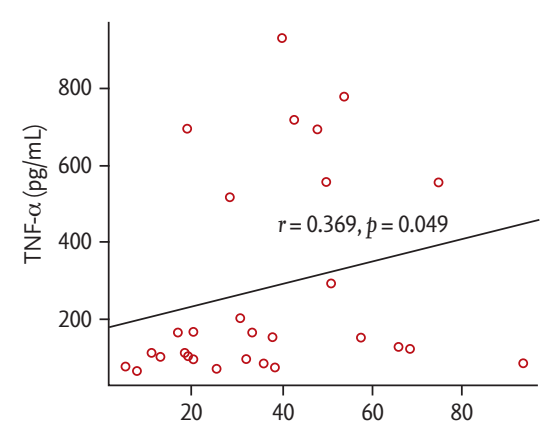

A

$\mathrm{IL}-17(\mathrm{pg} / \mathrm{mL})$

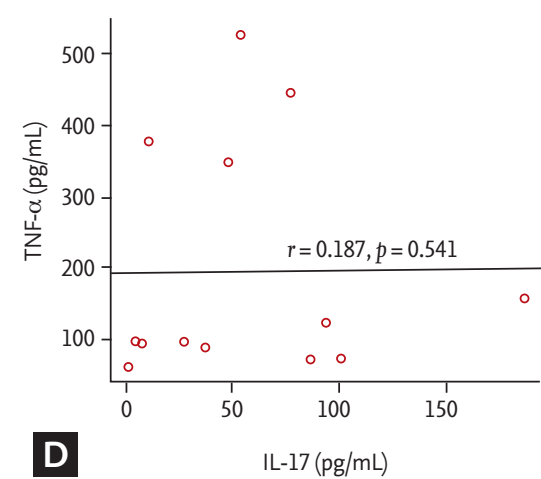

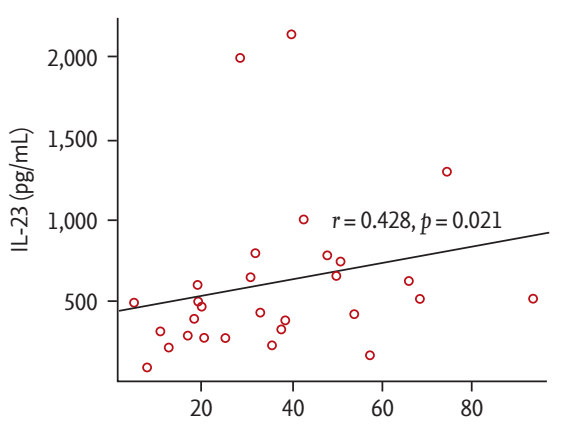

B

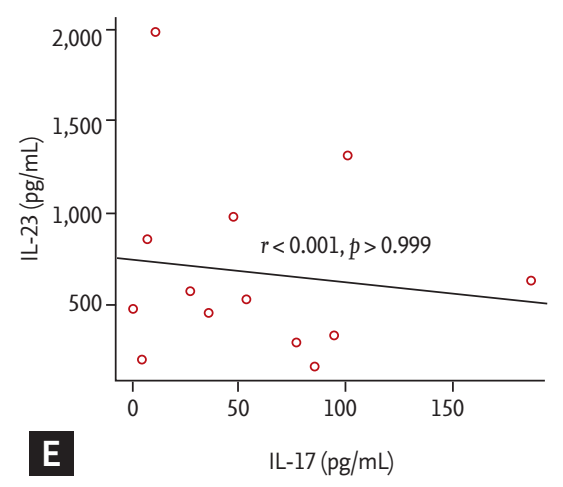

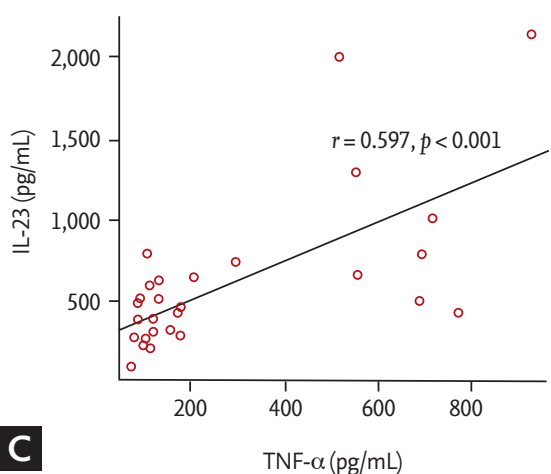

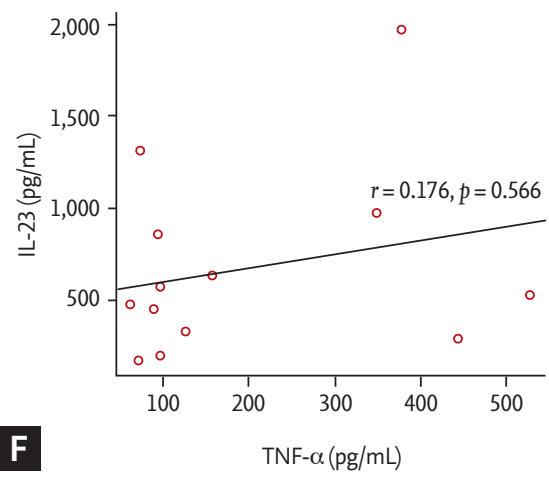

Figure 2. Correlations among (A, B, C) tumor necrosis factor- $\alpha$ (TNF- $\alpha$ ), interleukin 17 (IL-17), and IL-23 in the nonbiologics (n $=29)$ and $(\mathrm{D}, \mathrm{E}, \mathrm{F})$ biologics groups $(\mathrm{n}=13)$ in patients with ankylosing spondylitis.

biologics group in AS and RA. Comparing between AS and RA in each group revealed no significant difference in the cytokine levels.

\section{Correlation coefficients between cytokines accord- ing to the use of biologics in AS}

We investigated the correlation coefficients between cytokines in the non-biologics and biologics groups to see whether the use of biologics could alter the interactions between cytokines in AS (Fig. 2). The non-biologics group showed significant correlation between IL-17 and TNF- $\alpha(r=0.369)$, between IL-17 and IL-23 $(r=0.428)$, and between TNF- $\alpha$ and IL-23 $(r=0.597)$ (Fig. 2A-2C). However, there was no significant correlation between IL-17 and TNF- $\alpha(r=0.187)$, between IL-17 and IL-23 $(r=0)$, and between TNF- $\alpha$ and IL-23 $(r=0.176)$ (Fig. $2 \mathrm{D}-2 \mathrm{~F})$ in the biologics group. In patients with RA, there was a high correlation coefficients between cytokines in the nonbiologics group, but not in the biologics group (Supplementary Fig. 1).

\section{Correlation coefficients between disease activity and cytokines according to the use of biologics in AS}

The relationship between disease activity and cytokine in joint fluid was investigated. There appears to be a higher correlation between BASDAI and cytokine in the nonbiologics group than in the biologics group $(r$ $=0.428$ vs. $r=0.061$ in IL-17; $r=0.550$ vs. $\mathrm{r}=0$ in IL-23; and $r=0.426$ vs. $r=0.469$ in TNF- $\alpha$ ) (Supplementary Fig. 2). ESR and CRP did not show a significant association with cytokines (Supplementary Figs. 3 and 4), except that ESR and TNF- $\alpha$ were highly correlated in the biologics group $(r=0.022)$ (Supplementary Fig. $4 \mathrm{~F}$ ).

\section{Effect of DMARDs and biologics on the cytokine levels in the synovial fluid of patients with AS}

We subdivided AS patients according to the use of DMARDs and biologics to further demonstrate their effect in synovial fluid (Fig. 3). The level of TNF- $\alpha$ did not differ according to the use of DMARDs. However, the level of IL-17 was significantly higher in the group treat- 

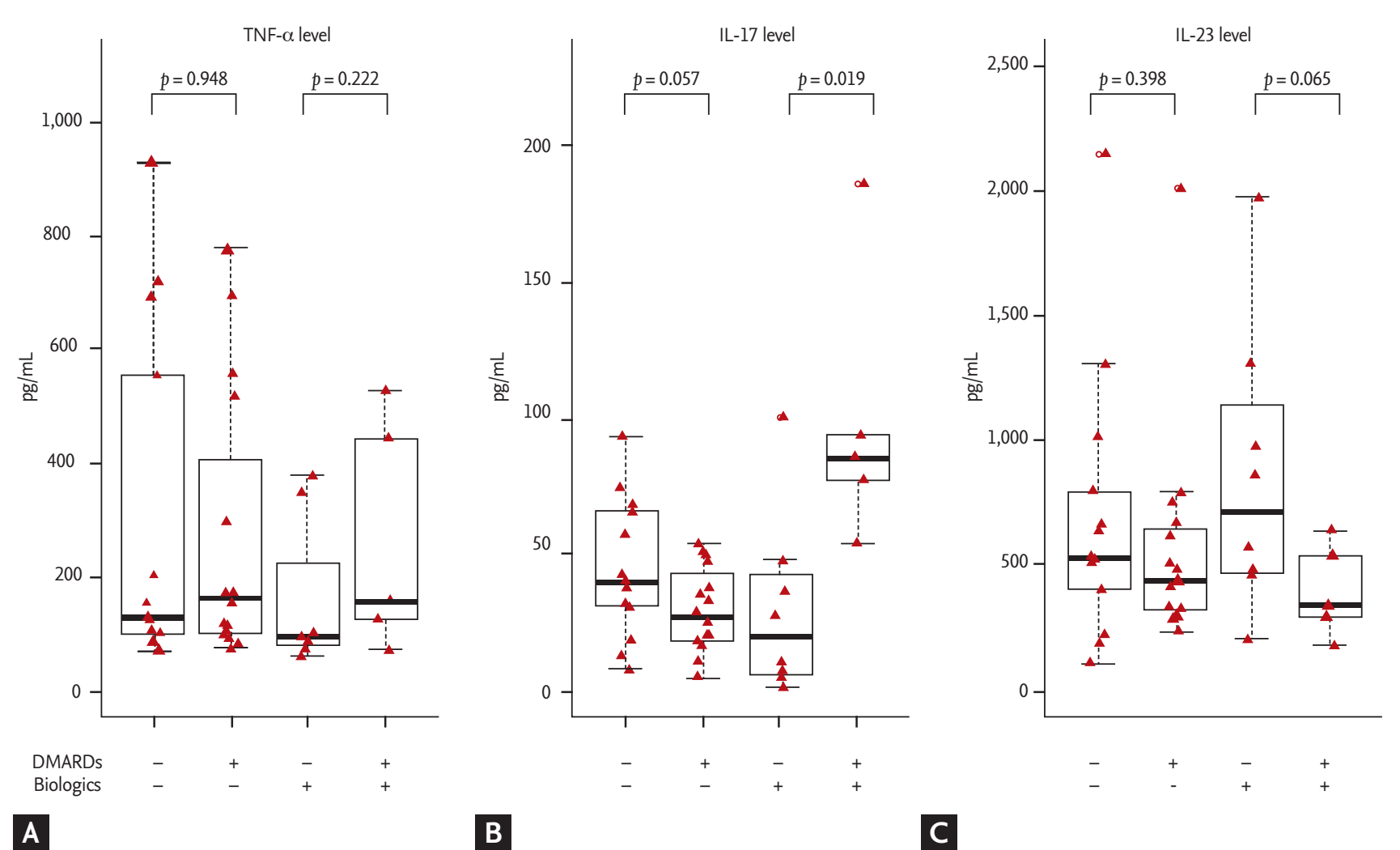

B

C

Figure 3. Differences in (A) tumor necrosis factor- $\alpha$ (TNF- $\alpha$ ), (B) interleukin 17 (IL-17), and (C) IL-23 levels according to the use of disease-modifying anti-rheumatic drugs (DMARDs) and biologics in patients with ankylosing spondylitis (without both DMARDs and biologics, $\mathrm{n}=13$; only with DMARDs, $\mathrm{n}=16$; only with biologics, $\mathrm{n}=8$; with both DMARDs and biologics, $\mathrm{n}=5$ ).

ed with both DMARDs and biologics compared with the group only with biologics $(p=0.019)$. Interestingly, five serial samples with high IL-17 level were from two patients with recurrent arthritis. In addition, although there was no significant difference, the level of IL-23 tend to be higher in the group treated only with biologics compared with the group treated with both DMARDs and biologics in the biologics group $(p=0.065)$.

\section{DISCUSSION}

We examined the levels of TNF- $\alpha$, IL-17, and IL-23 in the synovial fluid of patients with AS and RA and the effect of the drug on the levels of those cytokines. The cytokine profiles of the synovial fluid differed between AS and RA. Furthermore, we identified that the cytokine profile of the synovial fluid differs depending on the drug used in AS. In two patients with AS, despite treatment with TNF- $\alpha$ inhibitor, recurrent arthritis occurred and the relationship of cytokines in the synovial fluid was different from that of patients who did not receive TNF- $\alpha$ inhibitor treatment.

Compared with synovial fluids of various diseases, such as undifferentiated arthritis, juvenile arthritis, spondylitis, reactive arthritis, gout, and Behcet's disease, it was confirmed that various cytokines including TNF- $\alpha$ were increased in synovial fluid of RA [15]. This evidence showed that RA has been known to have complex cytokine networks in autoimmune processes. In addition, that study suggested that cytokine profiles in RA synovial fluid vary with treatment and response to therapy. Our study also suggested that the treatment of AS may change the cytokine profiles of the synovial fluid. However, those changes in AS was different from RA.

The IL-23/Th17 axis is an important pathologic mechanism, and polymorphisms in the receptor for IL-23 are associated with AS [16-19]. There was evidence that increased IL-23 could promote the expansion of Th17 cells and enhance the production of IL-17 and TNF- $\alpha$ in AS. 
In previous studies, the levels of IL-23 and IL-17 were increased in the serum of patients with AS [20-22], but it was also observed in patients with RA [7,23]. Additionally, there was a strong positive correlation between the serum levels of IL-17 and IL-23 in patient with AS [22]. The relationship between IL-23 and IL-17 appears to play an important role in inflammatory arthritis and has been implicated in several studies of spondyloarthritis (SpA) [21,22,24]. We observed the relationship between IL-23, IL-17, and TNF- $\alpha$ in synovial fluid in the pathogenesis of AS. Moreover, we also observed the profile changes of important cytokines according to the drugs because the effect of the treatment could also affect the level of cytokines synovial fluid [15].

Singh et al. $[24,25]$ measured the levels of several proinflammatory cytokines in the synovial fluid of patients with SpA. They suggested that inflammatory synovitis in reactive arthritis and undifferentiated $\mathrm{SpA}$ is mediated by proinflammatory cytokines such as IL-17, IL-6, IL-1b, and IL-21 and that there is good correlation among IL17, IL-6, and IL-1b [24]. They showed the level of IL-17 in the synovial fluid of patients with reactive arthritis and undifferentiated SpA, which was similar to the levels of patients with RA. In addition, those results showed that IL-23 was undetectable in all synovial samples. However, we identified IL-23 in the synovial fluid, and the level of IL-23 was similar in both AS and RA.

We measured the important cytokine profiles of the synovial fluid in patients taking biologics. Both DMARD and biologics could have a significant effect on the cytokine profiles of the synovial fluid in AS. Of these, five samples of two patients treated with both DMARDs and TNF- $\alpha$ inhibitor had elevated levels of IL-17. Since these two patients previously had recurrent arthritis despite various treatment and their serial IL-17 levels were persistently high during the treatment, we suspect that IL-17 may have played an important role in the onset of AS, perhaps from the beginning of symptom in these patients.

Noordenbos et al. [26] presented an interesting result on IL-17 expression in mast cells. They suggested the mast cells are the main IL-17-expressing cell population in SpA synovitis and their infiltration in the synovium is a primary feature, rather than a consequence, of synovial inflammation, as it is observed early in the disease and is not affected by TNF blockade. In our study, IL-17 increased in two patients despite treatment with TNF inhibitors, and this might be related to IL-17-expressing cell in SpA synovitis. If these patients had increased IL17 levels in the synovial fluid before treatment with TNF inhibitors and IL-17 is main cytokine in synovial inflammation, they could be a subgroup of SpA that does not respond to TNF inhibitors.

Another noteworthy group in AS is the group of patients with arthritis treated only with biologics and without DMARDs. The level of IL-23 in this group tended to be higher than the group treated with both biologics and DMARDs. There is a possibility that arthritis was caused by IL-23 or by a proinflammatory cytokine induced by IL-23 $[17,19]$. Hence, some patients with peripheral arthritis dominated by IL-23 in AS may be better treated with the addition of DMARDs or other biologics such as IL-23 inhibitors.

Many studies have not considered that the use of drugs can have a significant impact on the relationship of each cytokine not only in serum but also in the synovial fluid of patients with arthritis $[20,22,24,27]$. The cytokine level in the joint fluid may vary depending on the drug being used for treatment. Thus, when conducting experimental studies on synovial fluid, we should be aware that inflammatory substances in the synovial fluid are affected by drugs used for the treatment of arthritis.

Our study had several limitations. First, the sample size was small, especially the number of patients with peripheral arthritis who were treated with biologics. Very few patients with peripheral arthritis were being treated with biologics. Second, we could not measure the changes in the cytokine levels of the same patient over time because of the short study period. Third, we studied only the peripheral involvement in AS, which chiefly involves the axial spine.

This study showed the differences in the three major proinflammatory cytokines of the synovial fluid between AS and RA. TNF- $\alpha$, IL-23, and IL-17 were all related to each other in patients treated without biologics, and their cytokine profiles differed depending on the drug being currently used such as biologics in both AS and RA. Furthermore, a subgroup of AS occurring in patients treated with biologics, in whom arthritis occurs dominantly by IL-17 or IL-23, is suggested. 


\section{KEY MESSAGE}

1. The cytokine profiles of the synovial fluid differed between ankylosing spondylitis (AS) and rheumatoid arthritis (RA).

2. The cytokine profile of the synovial fluid may differ depending on the drug used in both AS and RA.

3. Interleukin (IL)-23 and IL-17 cytokine might be an important factor in some patients who are unresponsive to biologics in AS.

\section{Conflict of interest}

No potential conflict of interest relevant to this article was reported.

\section{Acknowledgments}

This study was supported by research fund from the Rheumatology Research Foundation (RRF-2015-01). This study was also supported by the Basic Science Research Program through the National Research Foundation of Korea (NRF) funded by the Ministry of Science, ICT and Future (2016R1A2B4008606), the Ministry of Education (2017R1A6A3A11034394), and a grant from the Korea Health Technology R\&D project through the Korea Health Industry Development Institute (KHIDI), funded by the Ministry of Health and Welfare, Republic of Korea (HI17Co888).

\section{REFERENCES}

1. McInnes IB, Schett G. Cytokines in the pathogenesis of rheumatoid arthritis. Nat Rev Immunol 2007;7:429-442.

2. Appel H, Loddenkemper C, Miossec P. Rheumatoid arthritis and ankylosing spondylitis: pathology of acute inflammation. Clin Exp Rheumatol 2009;27:S15-S19.

3. Smolen JS, Landewe R, Bijlsma J, et al. EULAR recommendations for the management of rheumatoid arthritis with synthetic and biological disease-modifying antirheumatic drugs: 2016 update. Ann Rheum Dis 2017;76:960-977.

4. van der Heijde D, Ramiro S, Landewe R, et al. 2016 Update of the ASAS-EULAR management recommendations for axial spondyloarthritis. Ann Rheum Dis 2017;76:978-
991.

5. Baeten D, Kruithof E, Van den Bosch F, et al. Immunomodulatory effects of anti-tumor necrosis factor alpha therapy on synovium in spondylarthropathy: histologic findings in eight patients from an open-label pilot study. Arthritis Rheum 2001;44:186-195.

6. Feldmann M. Development of anti-TNF therapy for rheumatoid arthritis. Nat Rev Immunol 2002;2:364-371.

7. Raza K, Falciani F, Curnow SJ, et al. Early rheumatoid arthritis is characterized by a distinct and transient synovial fluid cytokine profile of $\mathrm{T}$ cell and stromal cell origin. Arthritis Res Ther 2005;7:R784-R795.

8. Braun J, Baraliakos X, Deodhar A, et al. Effect of secukinumab on clinical and radiographic outcomes in ankylosing spondylitis: 2-year results from the randomised phase III MEASURE 1 study. Ann Rheum Dis 2017;76:1070-1077.

9. Coates LC, Cawkwell LS, Ng NW, et al. Real life experience confirms sustained response to long-term biologics and switching in ankylosing spondylitis. Rheumatology 2008;47:897-900.

10. Deodhar A, Yu D. Switching tumor necrosis factor inhibitors in the treatment of axial spondyloarthritis. Semin Arthritis Rheum 2017;47:343-350.

11. Kiltz U, Heldmann F, Baraliakos X, Braun J. Treatment of ankylosing spondylitis in patients refractory to TNF-inhibition: are there alternatives? Curr Opin Rheumatol 2012;24:252-260.

12. Aletaha D, Neogi T, Silman AJ, et al. 2010 Rheumatoid arthritis classification criteria: an American College of Rheumatology/European League Against Rheumatism collaborative initiative. Ann Rheum Dis 2010;69:15801588.

13. van der Linden S, Valkenburg HA, Cats A. Evaluation of diagnostic criteria for ankylosing spondylitis. A proposal for modification of the New York criteria. Arthritis Rheum 1984;27:361-368.

14. Calin A, Garrett S, Whitelock H, et al. A new approach to defining functional ability in ankylosing spondylitis: the development of the Bath Ankylosing Spondylitis Functional Index. J Rheumatol 1994;21:2281-2285.

15. Wright HL, Bucknall RC, Moots RJ, Edwards SW. Analysis of SF and plasma cytokines provides insights into the mechanisms of inflammatory arthritis and may predict response to therapy. Rheumatology 2012;51:451-459.

16. Australo-Anglo-American Spondyloarthritis Consortium 
(TASC), Reveille JD, Sims AM, et al. Genome-wide association study of ankylosing spondylitis identifies non-MHC susceptibility loci. Nat Genet 2010;42:123-127.

17. Harrington LE, Hatton RD, Mangan PR, et al. Interleukin 17-producing $\mathrm{CD}_{4}+$ effector $\mathrm{T}$ cells develop via a lineage distinct from the $\mathrm{T}$ helper type 1 and 2 lineages. Nat Immunol 2005;6:1123-1132.

18. Lories RJ, McInnes IB. Primed for inflammation: enthesis-resident T cells. Nat Med 2012;18:1018-1019.

19. Lubberts E. The IL-23-IL-17 axis in inflammatory arthritis. Nat Rev Rheumatol 2015;11:562.

20. Wendling D, Cedoz JP, Racadot E, Dumoulin G. Serum IL-17, BMP-7, and bone turnover markers in patients with ankylosing spondylitis. Joint Bone Spine 2007;74:304-305.

21. Wang X, Lin Z, Wei Q, Jiang Y, Gu J. Expression of IL-23 and IL-17 and effect of IL-23 on IL-17 production in ankylosing spondylitis. Rheumatol Int 2009;29:1343-1347.

22. Mei Y, Pan F, Gao J, et al. Increased serum IL-17 and IL-23 in the patient with ankylosing spondylitis. Clin Rheuma- tol 2011;30:269-273.

23. Ziolkowska M, Koc A, Luszczykiewicz G, et al. High levels of IL-17 in rheumatoid arthritis patients: IL-15 triggers in vitro IL-17 production via cyclosporin A-sensitive mechanism. J Immunol 2000;164:2832-2838.

24. Singh AK, Misra R, Aggarwal A. Th-17 associated cytokines in patients with reactive arthritis/undifferentiated spondyloarthropathy. Clin Rheumatol 2011;30:771-776.

25. Singh R, Aggarwal A, Misra R. Th1/Th17 cytokine profiles in patients with reactive arthritis/undifferentiated spondyloarthropathy. J Rheumatol 2007;34:2285-2290.

26. Noordenbos T, Yeremenko N, Gofita I, et al. Interleukin-17-positive mast cells contribute to synovial inflammation in spondylarthritis. Arthritis Rheum 2012;64:99109.

27. Singh JA, Saag KG, Bridges SL Jr, et al. 2015 American College of Rheumatology guideline for the treatment of rheumatoid arthritis. Arthritis Rheumatol 2016;68:1-26. 

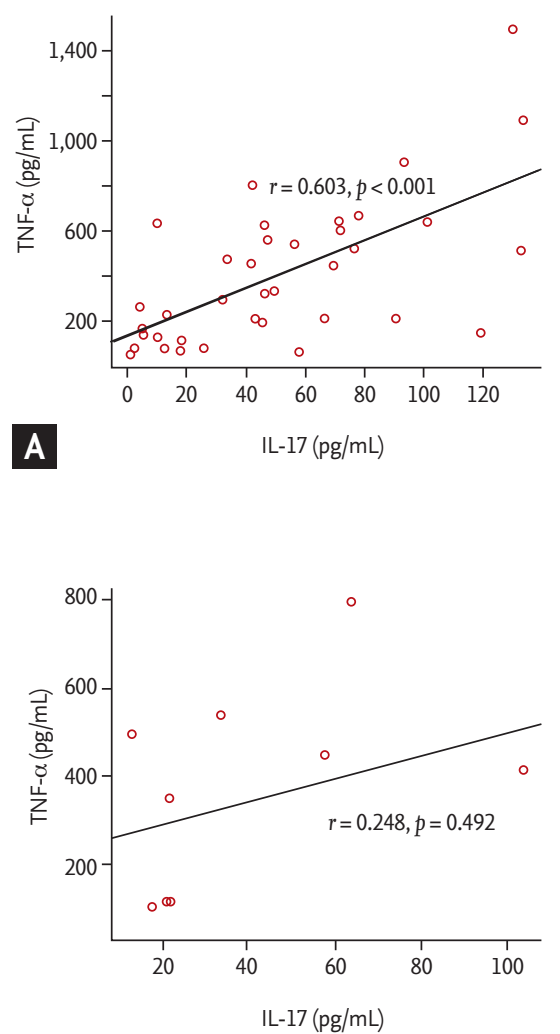

D

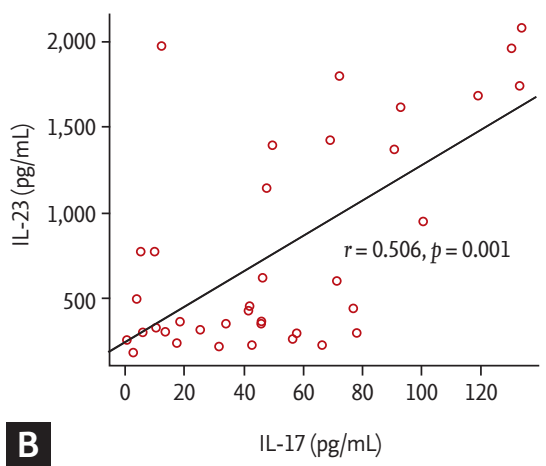

B

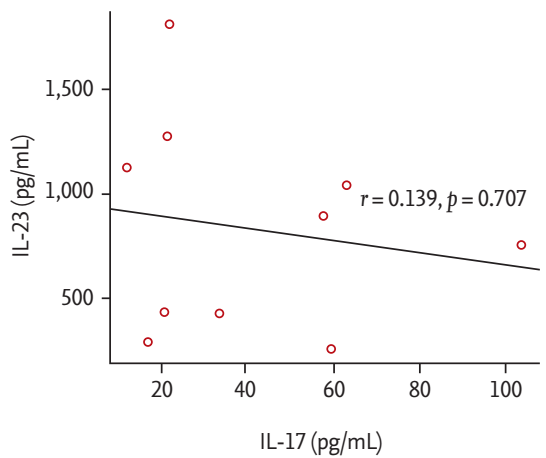

E
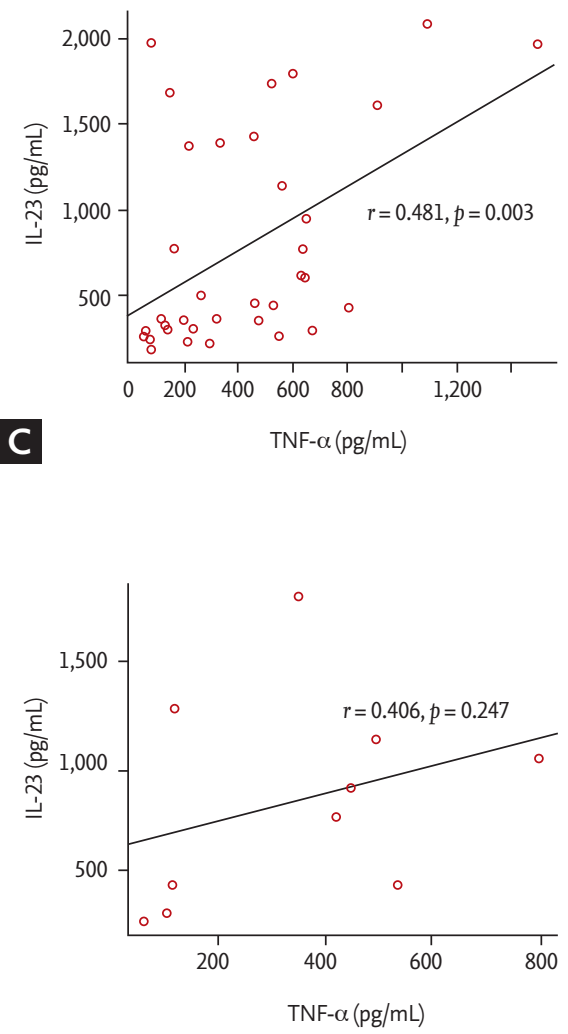

$\mathbf{F}$

Supplementary Figure 1. Correlations among (A, B, C) tumor necrosis factor- $\alpha$ (TNF- $\alpha$ ), interleukin 17 (IL-17), and IL-23 in the nonbiologics $(n=37)$ and $(D, E, F)$ biologics groups $(n=10)$ in patients with rheumatoid arthritis. 


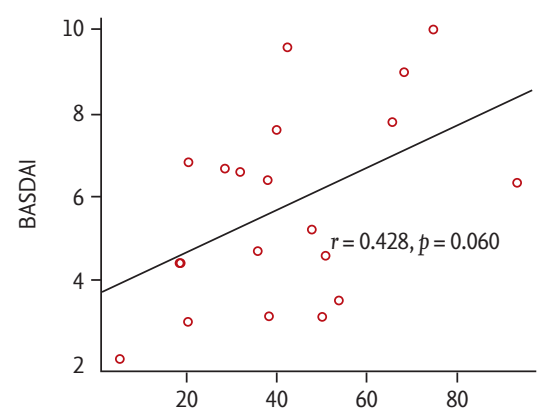

A

$\mathrm{IL}-17(\mathrm{pg} / \mathrm{mL})$

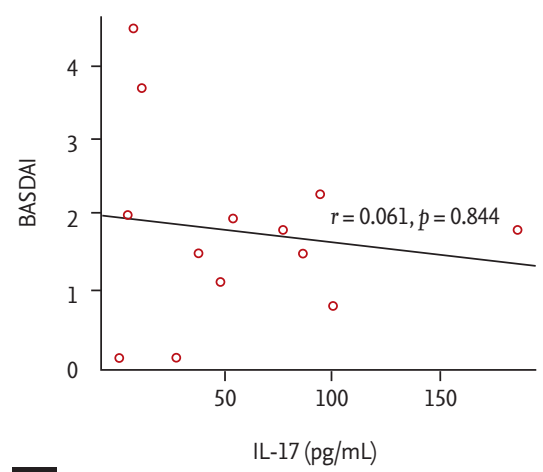

D

Supplementary Figure 2. Correlations between the Bath Ankylosing Spondylitis Disease Activity Index (BASDAI) and (A, B, C) cytokines in the nonbiologics $(n=29)$ and $(D, E, F)$ biologics groups $(n=13)$ in patients with ankylosing spondylitis. IL, interleukin; TNF- $\alpha$, tumor necrosis factor- $\alpha$.
B

$\mathrm{IL}-23(\mathrm{pg} / \mathrm{mL})$

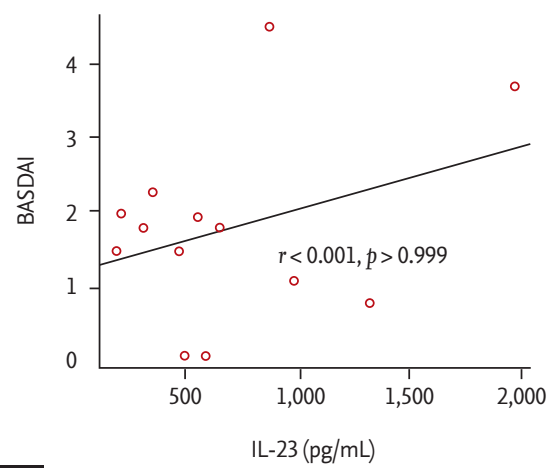

E

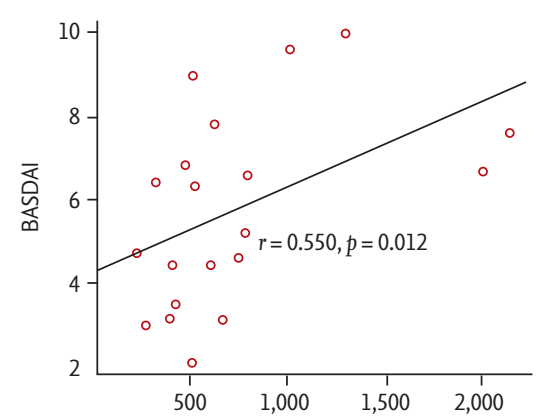

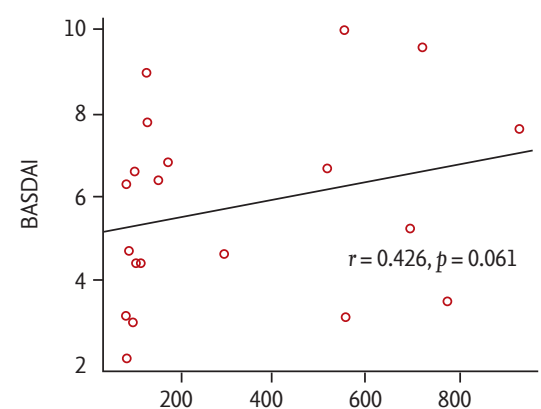

C

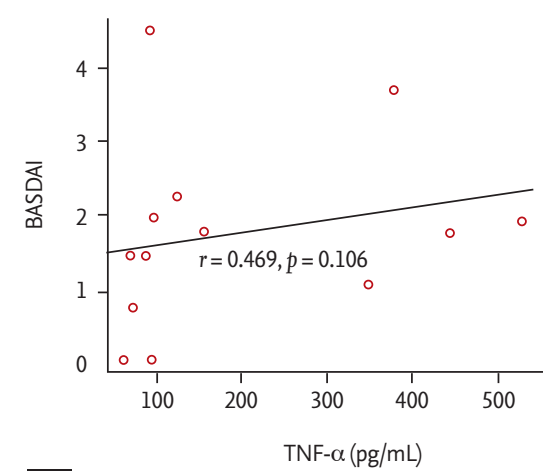

F 


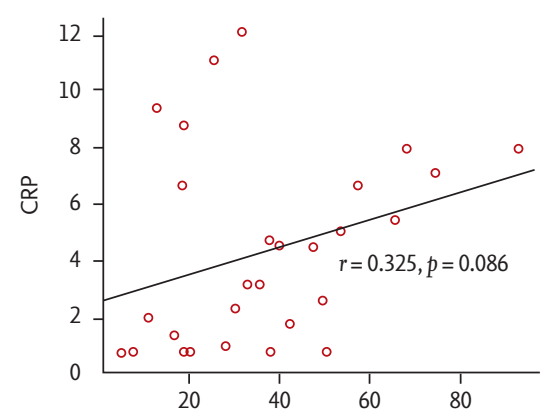

A

$\mathrm{IL}-17(\mathrm{pg} / \mathrm{mL})$

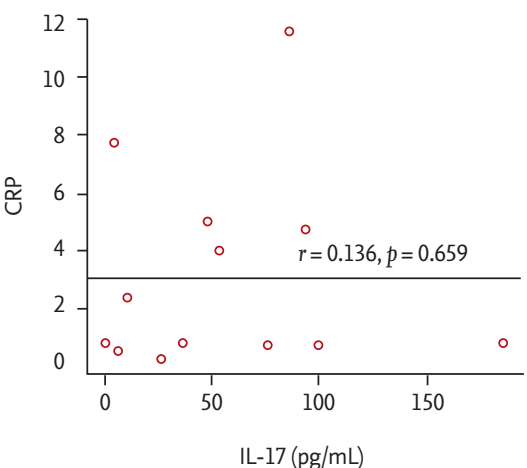

D

Supplementary Figure 3. Correlations between C-reactive protein (CRP) and (A, B, C) cytokines in the nonbiologics (n = 29) and $(\mathrm{D}, \mathrm{E}, \mathrm{F})$ biologics groups $(\mathrm{n}=13)$ in patients with ankylosing spondylitis. IL, interleukin; TNF- $\alpha$, tumor necrosis factor- $\alpha$.

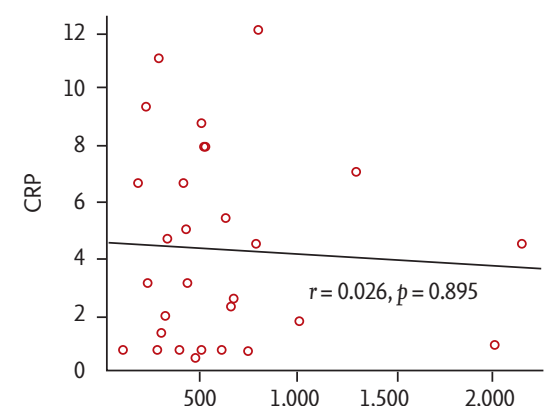

B

$\mathrm{IL}-23(\mathrm{pg} / \mathrm{mL})$

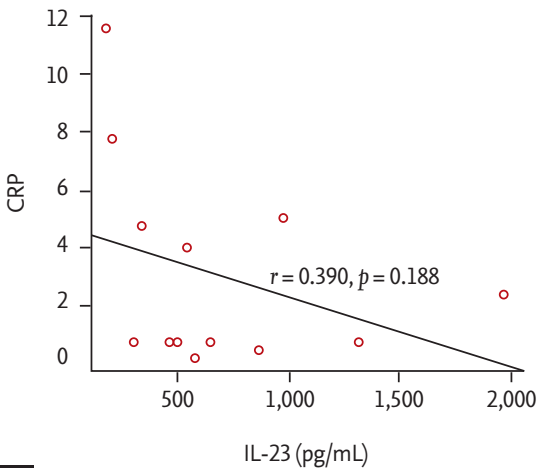

E

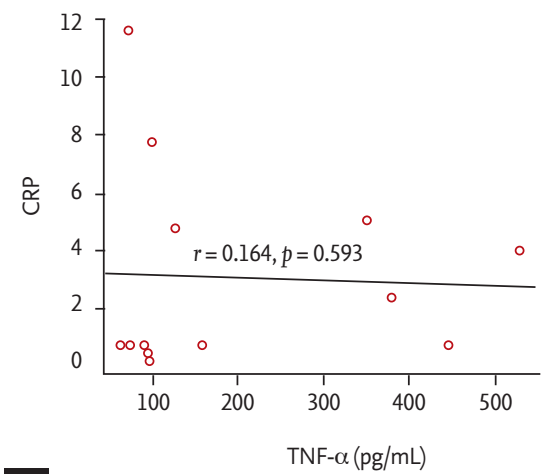

$\mathbf{F}$

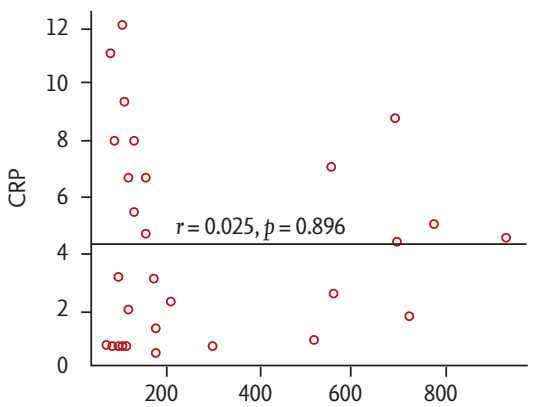

C

$\mathrm{TNF}-\alpha(\mathrm{pg} / \mathrm{mL})$ 


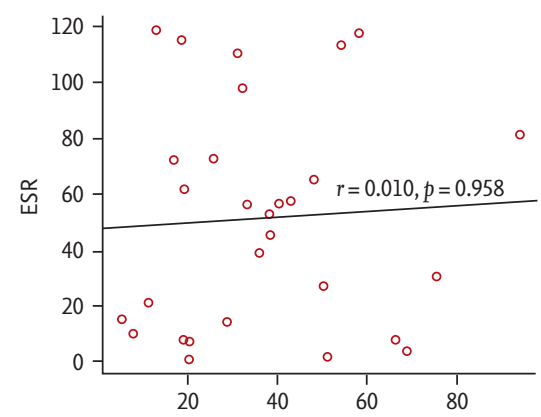

A

$\mathrm{IL}-17(\mathrm{pg} / \mathrm{mL})$

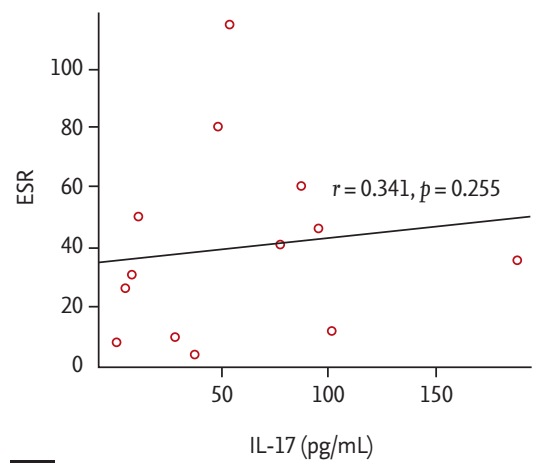

D

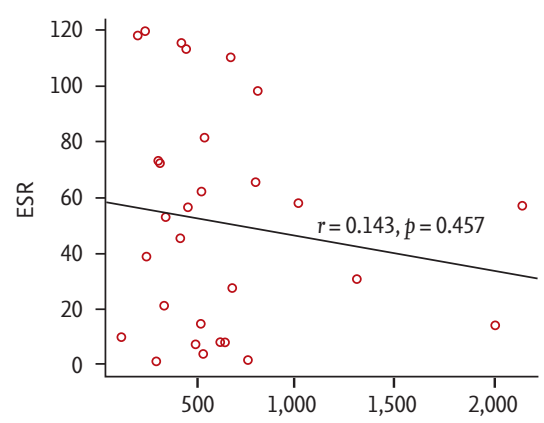

B

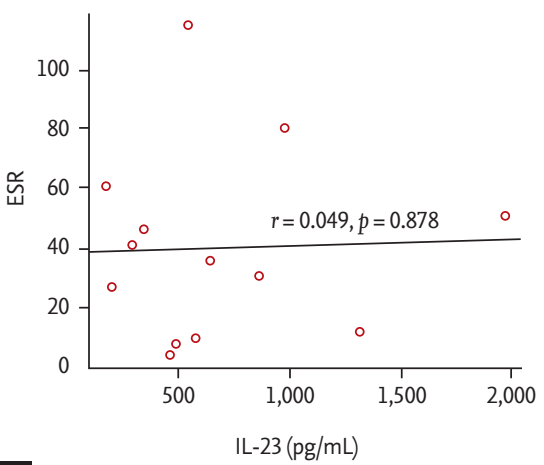

E

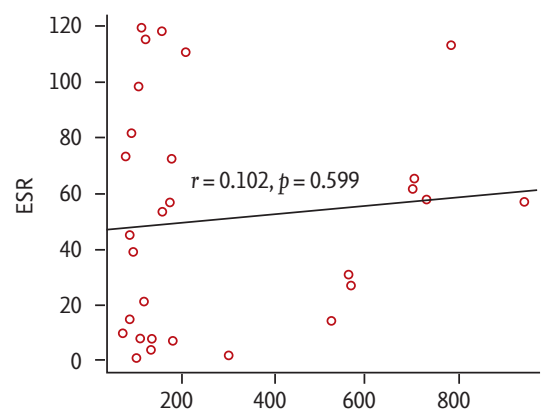

C $\quad$ TNF- $\alpha(\mathrm{pg} / \mathrm{mL})$

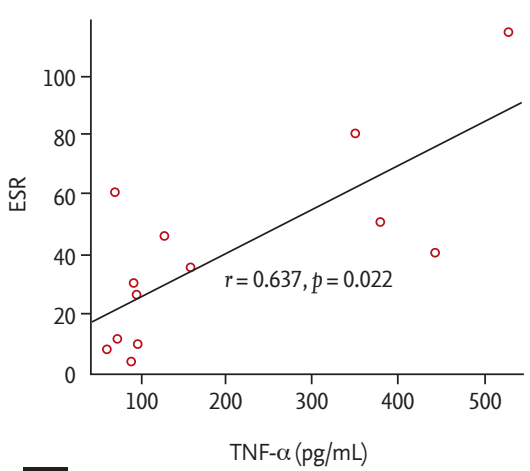

$\mathbf{F}$

Supplementary Figure 4. Correlations between erythrocyte sedimentation ratio (ESR) and (A, B, C) cytokines in the nonbiolog$\operatorname{ics}(n=29)$ and $(D, E, F)$ biologics groups $(n=13)$ in patients with ankylosing spondylitis. IL, interleukin; TNF- $\alpha$, tumor necrosis factor- $\alpha$. 